\title{
Matrix-assisted laser desorption/ionization time- of-flight mass spectrometry-based identification of security-sensitive bacteria: Considerations for Canadian Bruker users
}

\author{
Kym Antonation ${ }^{1 \star}$, Dobryan Tracz ${ }^{1}$, Ashley Dreger ${ }^{1}$, Cindi Corbett ${ }^{1,2}$
}

\begin{abstract}
Background: The use of matrix-assisted laser desorption/ionization time-of-flight (MALDI-TOF) mass spectrometry (MS) systems for bacterial identification has rapidly become a front line tool for diagnostic laboratories, superseding classical microbiological methods that previously triggered the identification of higher risk pathogens. Unknown Risk Group 3 isolates have been misidentified as less pathogenic species due to spectral library availability, content and quality. Consequently, exposure to higher risk pathogens has been reported within Canadian laboratory staff following the implementation of MALDI-TOF MS. This overview aims to communicate the potential risk to laboratory staff of inaccurate identification of security-sensitive biological agents (SSBA) bacteria and to provide suggestions to mitigate.
\end{abstract}

Methods: Cultures were manipulated in a Biosafety Level 3 laboratory, prepared for MALDI-TOF MS analysis via full chemical extraction and analysed on a Bruker Microflex LT instrument. Data were analyzed with Biotyper software; comparing raw spectra against MS profiles in three libraries: Bruker Taxonomy; Bruker Security-Restricted; and National Microbiology Laboratory (NML) SSBA libraries. Four years of Bruker MALDI-TOF MS data acquired in-house were reviewed.

Results: In general, the Bruker MS spectral libraries were less successful in identifying the SSBA bacteria. More successful was the NML library. For example, using a high score cut-off (greater than 2.0), the Bruker SR library was unable to identify $52.8 \%$ of our Risk Group 3 agents and near neighbours to the species-level with confidence, whereas the custom NML library was unable to identify only $20.3 \%$ of the samples.

Conclusion: The last four years of data demonstrated both the importance of library selection and the limitations of the various spectral libraries. Enhanced standard operating procedures are advised to reduce laboratory exposure to SSBAs when using MALDI-TOF MS as a front line identification tool.
This work is licensed under a Creative Commons Attribution 4.0 International License.

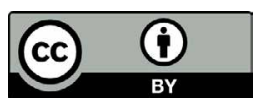

Affiliations

${ }^{1}$ National Microbiology Laboratory, Public Health Agency of Canada, Winnipeg, MB

2 University of Manitoba, Department of Medical Microbiology and Infectious Diseases, Winnipeg, MB

\section{${ }^{\star}$ Correspondence:}

kym.antonation@canada.ca

Suggested citation: Antonation KS, Tracz DM, Dreger AD, Corbett CR. Matrix-assisted laser

desorption/ionization time-of-flight mass spectrometry-based identification of security-sensitive bacteria:

Considerations for Canadian Bruker users. Can Commun Dis Rep 2020;46(10):333-8.

https://doi.org/10.14745/ccdr.v46i10a04

Keywords: MALDI-TOF mass spectrometry, bacterial pathogens, clinical microbiology laboratory diagnostics, security-sensitive

\section{Introduction}

Within the last decade, clinical microbiology laboratories have moved towards replacing traditional biochemical-based techniques with new matrix-assisted laser desorption/ionization time-of-flight (MALDI-TOF) mass spectrometry (MS) systems for bacterial identification $(1,2)$. As a rapid, low-cost, straight-forward, and high-throughput method, MALDI-TOF MS is a powerful tool for bacterial diagnostics and has led to significant cost savings and improved efficiencies in laboratories 
$(3,4)$. However, the movement away from classical bacterial testing methods to unilateral use of MALDI-TOF MS presents a very real hazard for clinical laboratories. Despite cautionary tales in the open literature, many clinical laboratories prepare MALDI-TOF MS target plates with live bacterial culture on an open laboratory bench in a Biosafety Level 2 (BSL-2) laboratory area, leading to inadvertent exposure(s) with Risk Group 3 (RG3) agents that may have found their way through the laboratory (5-7).

Within Canada, five exposures to RG3 agents reported within a single nine-month period prompted a root cause investigation of the technology and its use (8). Su et al. found that between 2015 and 2017, eight incidents with 39 exposures were reported as a result of misidentification of RG3 bacteria via the use of commercial MALDI-TOF MS systems and associated libraries (8). Brucella species, Francisella tularensis and Burkholderia pseudomallei made up the bulk of these exposures. While the current Association of Public Health Laboratories (APHL) sentinel guidelines clearly define expectations when working with a possible RG3 agent, there are situations where unknown isolates fall into the laboratory workflow that may be RG3. These unknown bacteria can be misidentified as less pathogenic near neighbour species due to 1) the contents of the spectral library supplied with the MS instrument or 2) the quality of the bacterial sample (9-11).

Certainly, many of the security-sensitive bacteria do not have reference spectra in the library supplied with the MS instrument, and in the absence of reference spectra for these species, the tool will either produce no identification (as in the case with Brucella spp.) or provide the identity of a closely related organism. We have observed that identification scores provided for a $B$. anthracis misidentified as a $B$. cereus can be quite high (i.e. greater than 2.0; considered high confidence identification), causing no suspicion on the part of the clinician that misidentification has occurred until further testing is complete, prolonging the possible exposure period.

Further, the 2018 Canada Communicable Disease Report indicated that some laboratorians did not know which reference library they were using for identification (8). Assuming that this implied a lack of understanding in the content and confidence of the identification library, we re-examined the last four years of MALDI-TOF MS spectra derived from well-characterized or reference strains of security-relevant bacteria and near neighbours that were obtained in-house to bring awareness of the diagnostic sensitivity and specificity of MALDI-TOF MS libraries and considerations for their use. The laboratory at the Public Health Agency of Canada's National Microbiology Laboratory (NML) focusses on security-sensitive biological agents (SSBAs), and is the gatekeeper for the Canadian Laboratory Response Network. As such, we have previously reported on both the safety of MALDI-TOF MS preparation methods and the sensitivity of the libraries for identification of the bacterial SSBAs
(12-14). Reviewing the last four years of data demonstrated both the importance of library selection and the limitations of the various libraries.

\section{Methods}

Cultures were manipulated in a Biosafety Level 3 (BSL-3) laboratory, prepared for MALDI-TOF MS analysis via full chemical extractions (70\% ethanol-70\% formic acid-acetonitrile) and brought to a Bruker Microflex LT instrument (Bruker Daltronics) housed in BSL-2 laboratory, as previously described (13). FlexControl software (version 3.4, build 135) acquired spectra based on 500 individual laser shots of four independent spots per sample.

Data were analyzed with Biotyper software (ver 3.1, build 66), searching raw spectra against bacterial mass spectral profiles (MSP) in the following libraries: 1) the Bruker Taxonomy library ( $n=5,989$ MSPs, not containing SSBAs); 2 ) the Bruker Security-Restricted (SR) library ( $n=123$ MSPs, containing SSBAs); and 3) a locally-developed NML SSBA library ( $n=121$ MSPs, containing both SSBAs and near neighbour MSPs). In addition, the NML curated library contains high-quality MSPs that exceed the content of the Bruker SR library for B. anthracis, Yersinia pestis, F. tularensis and the Brucella species (11).

The top four Biotyper software MSP matches and their associated match score were recorded for each of four spots per bacterial sample to comprise the sample population. This was more representative of the sample distribution than choosing only the top single match per spot. Identification at the "secure genus identification, probable species identification" level (greater than 2.0 match score) was used for all comparative calculations throughout unless detailed otherwise. Diagnostic sensitivity and specificity were calculated for each SSBA against its near neighbour species, based on the greater than 2.0 match score threshold: $B$. anthracis ( $n=240$ sample spots) versus other $B$. cereus complex species $(n=256) ;$ Y. pestis $(n=272)$ vs. Y. pseudotuberculosis $(n=160) ; F$. tularensis $(n=528)$ vs. other Francisella $(n=48)$. Brucella spp. $(n=816)$ have no near neighbour.

\section{Results and discussion}

Since the standard Bruker Taxonomy library does not contain any highly-pathogenic SSBA entries, the sensitivity for all SSBA samples using only the standard proprietary Bruker library is $0 \%$. Laboratories that have access only to the Bruker library should consider obtaining additional libraries that contain SSBA spectral profiles and/or utilizing enhanced standard operating procedures to recognize a potential exposure threat (as described below). Only clinical laboratories that have access to the specialized Bruker SR library or to the NML SSBA library can identify 
highly-pathogenic bacteria with MALDI-TOF MS technology at this time, with varying levels of confidence (as described below).

\section{Yersinia pestis}

The MALDI-TOF MS analysis of 17 Y. pestis isolates $(n=272$ total identification results) and $10 \mathrm{Y}$. pseudotuberculosis isolates ( $n=160$ identification results) yielded a sensitivity of $41.9 \%$ and specificity of $93.1 \%$ using the Bruker Taxonomy and SR library together (Table 1). In comparison, improved results for $Y$. pestis identification were obtained when using the NML SSBA library, which yielded a sensitivity of $70.6 \%$.

\section{Table 1: Sensitivity and specificity values derived from MALDI-TOF MS diagnostic test identification of security-sensitive biological agent bacteria at the National Microbiology Laboratory (2014-2018)}

\begin{tabular}{|l|r|r|r|r|r|r|}
\hline & \multirow{2}{*}{$\begin{array}{c}\text { SSBA } \\
\text { bacteria }\end{array}$} & \multirow{2}{*}{$\begin{array}{c}\text { Target } \\
\text { sample } \\
\text { size }\end{array}$} & $\begin{array}{c}\text { Non-target } \\
\text { (near } \\
\text { neighbour) } \\
\text { sample size }\end{array}$ & \multicolumn{2}{|c|}{$\begin{array}{c}\text { Sensitivity of } \\
\text { database }\end{array}$} & \multicolumn{2}{|c|}{$\begin{array}{c}\text { Specificity of } \\
\text { database }\end{array}$} \\
\cline { 5 - 7 } & 272 & 160 & $41.9 \%$ & $70.6 \%$ & $93.1 \%$ & $86.9 \%$ \\
\hline $\begin{array}{l}\text { Yersinia } \\
\text { pestis }\end{array}$ & 528 & 48 & $32.2 \%$ & $77.5 \%$ & $100.0 \%$ & $100.0 \%$ \\
\hline $\begin{array}{l}\text { Francisella } \\
\text { tularensis }\end{array}$ & 240 & 256 & $86.3 \%$ & $90.4 \%$ & $80.5 \%$ & $98.8 \%$ \\
\hline $\begin{array}{l}\text { Bacillus } \\
\text { anthracis }\end{array}$ & 240 & NML & Bruker & NML \\
\hline
\end{tabular}

Abbreviations: MALDI-TOF MS, matrix-assisted laser desorption/ionization time-of-flight mass spectrometry; NML, National Microbiology Laboratory; SSBA, security-sensitive biological agent Note: Values were calculated using sample identification results that had a match score of greater than 2.0, reflecting high confidence species-level identification. Sample size is comprised of top four identification results obtained from four spots per isolate

Even with the enhanced SSBA library, 80 of the 272 identification results did not identify $Y$. pestis correctly, but rather produced a $Y$. pseudotuberculosis identification $(n=68)$ or, alternatively, allowed identification only to the genus-level. Indeed, these two species are genetically similar $(15,16)$, which has resulted in the diagnostic misidentification that has been reported in the literature (17) and demonstrated with this data set. However, despite their relatedness, MALDI-TOF MS differentiation can be accomplished via a single biomarker peak at $\mathrm{m} / \mathrm{z} 3065$, which is associated with the plasmid-encoded protein $\mathrm{Pla}$, as reported by Lasch et al. (18). Analysis of representative NML samples determined 65 of the 68 aforementioned Y. pestis misidentified as Y. pseudotuberculosis did have the $\mathrm{m} / \mathrm{z} 3065$ peak, which resulted in an increase in sensitivity of $95.6 \%$. No Y. pseudotuberculosis spectral profile demonstrated this peak, for a specificity of $100 \%$. Using this peak alone for Y. pestis differentiation is superior to both the results seen with the Bruker library and the NML's SSBA library.

Nevertheless, with or without an augmented library, laboratory staff should be aware that a top match of $Y$. pseudotuberculosis could actually indicate the presence of a $Y$. pestis isolate. In addition, small gram-negative isolates with characteristic safety pin staining and "fried egg" colony morphology should immediately cause reversion to BSL-3 practices in a BSL-2 environment and follow APHL sentinel guidelines to rule-out or refer-out.

\section{Francisella tularensis}

Analysis of 33 known F. tularensis isolates (528 total identification results) determined a sensitivity rate of $32.2 \%$ using the Bruker Taxonomy and SR library (Table 1). Again, using the NML SSBA library, sensitivity was higher at $77.5 \%$. Both gave a specificity of $100 \%$; therefore, while a specimen can be falsely negative for F. tularensis, a positive identification of $F$. tularensis is certain. This is corroborated by a previous study by Seibold et al. (19), which found a Bruker library supplemented with Francisella spp. MSPs correctly identified $100 \%$ of Francisella isolates $(n=45)$ to the species-level. Further, in this review, we found that achieving a secure genus, probable species identification (match score greater than 2.0) for F. tularensis was difficult. Many F. tularensis isolates were identified as such within the match score range of 1.7-2.0 (i.e. probable genus identification). A much greater percentage of identification results reached the higher confidence species-level score using the NML SSBA library rather than the Bruker SR library $(77.5 \%$ vs. $32.2 \%$ had scores greater than 2.0), and a lesser proportion was unidentifiable entirely ( $8 \%$ vs. $19 \%$ ), which reflects library reference spectral quality and quantity.

Although F. tularensis has no near neighbours that are as close as the Y. pestis/Y. pseudotuberculosis relationship, any Francisella genus-level identification using MALDI-TOF MS should cause immediate concern, especially when considered with the morphological observation of tiny gram-negative bacilli that are slow growing and show preference to media supplemented with cysteine.

\section{Bacillus anthracis}

When comparing cultures of the Bacillus cereus complex, MALDI-TOF MS showed high sensitivity for detecting B. anthracis, but the Bruker libraries provided lower specificity than the NML's SSBA library (Table 1). Analysis of 15 known $B$. anthracis isolates ( $n=240$ results) and 16 non- $B$. anthracis/B. cereus complex species ( $n=256$ results) found both the Bruker SR library and the NML SSBA library had high sensitivity ( $86.3 \%$ and $90.4 \%$, respectively), but the Bruker library was markedly lower in specificity than NML ( $80.5 \%$ and $98.8 \%$, respectively). Both false positives and false negatives are possible, even with an augmented library, and an identification of any member of the $B$. cereus complex should stimulate awareness. Laboratories should also be aware of distinguishing features of $B$. anthracis: large $(10 \mu \mathrm{M})$ gram positive, spore forming rods that exhibit ground glass colonies that are non-hemolytic and catalase positive. B. cereus biovar anthracis isolates have exhibited motility, thereby eliminating this characteristic as a tool for rule-out (20). 


\section{Brucella species}

The standard Bruker taxonomy library does not contain Brucella spp., and the Bruker SR library contains only B. melitensis. Thus, the sensitivity was $0 \%$ for non- $B$. melitensis species of Brucella (B. abortus, B. canis, B. ovis, B. suis). The Bruker SR library test sensitivity for $B$. melitensis $(n=560)$ was $83.2 \%$, with notably no other possible Brucella matches in that library. More informatively, Brucella spp. isolates were correctly-identified at the genus-level in $99.6 \%$ instances with the SR library and $100 \%$ with the NML-SSBA library. The power of the NML enhanced library is within species-level identification; here, individual Brucella species were identified (Table 2) with varied levels of sensitivity (48.8\% to $88.4 \%$ ), but with higher levels of specificity (82.8\% to $99.3 \%$ ). Ferreira et al. tested Brucella strains ( $n=131)$ against a MALDI-TOF MS library supplemented with Brucella MSPs, and found $100 \%$ correlation at a genuslevel, but varying degrees of species-level identification (e.g. B. abortus at $82.4 \%, B$. melitensis at $10.7 \%$ ) (21). Using a custommade MALDI-TOF library of 18 unique Brucella genotypes, Lista et al. correctly identified $98 \%$ of Brucella isolates $(n=152)$ to the species-level (22). Other studies report MALDI-TOF MS species-level identification of Brucella isolates at an accuracy of $92 \%(23)$ and $97 \%(24)$.

\section{Table 2: MALDI-TOF MS identification of Brucella species with the National Microbiology Laboratory-developed SSBA library}

\begin{tabular}{|l|r|r|}
\hline \multicolumn{1}{|c|}{ Brucella species } & Sensitivity (\%) & \multicolumn{1}{c|}{ Specificity (\%) } \\
\hline B. melitensis & 88.4 & 82.8 \\
\hline B. abortus & 53.1 & 96.0 \\
\hline B. canis & 56.3 & 90.5 \\
\hline B. ovis & 56.3 & 99.3 \\
\hline B. suis & 48.8 & 98.4 \\
\hline
\end{tabular}

Abbreviations: MALDI-TOF MS, matrix-assisted laser desorption/ionization time-of-flight mass spectrometry; SSBA, security-sensitive biological agent

Note: The proprietary Bruker library only contains B. melitensis profiles and no other Brucella species

Augmenting a MALDI-TOF MS library with Brucella spp. spectra would be the most appreciable gain for clinical laboratories due to the lack of near neighbours, and consequently, no identification or near neighbour trigger provided by the device. Regardless, labs should be aware of tiny gram-negative coccobacilli that stain weakly, grow slowly on chocolate media, and produce small, glistening colonies.

\section{Conclusion}

An understanding of the device library limitations and the application of enhanced standard operating procedures are key requirements for clinical laboratories using MALDI-TOF MS as their primary bacterial identification method. Previous studies have cited the importance of supplementing proprietary libraries with local, in-house developed profiles for bacterial identification $(9,11,25,26)$ and our data supports this for the
Bruker MALDI-TOF MS. Custom MALDI-TOF MS libraries improve identifications, thereby limiting misidentification of high consequence SSBA and subsequent laboratory exposures and incorrect diagnosis. Comparing the overall sensitivity of the Bruker MALDI-TOF MS to identify security-sensitive bacteria (e.g. $B$. anthracis, Y. pestis or F. tularensis), using the different libraries, we found an improvement from 47.2\% (Bruker Taxonomy and SR library) to $79.7 \%$ (NML SSBA library, inclusive of the Y. pestis biomarker peak analysis). If we disregard the rigid cut-off (using only match scores greater than 2.0) these values increase to $75 \%$ and $92.9 \%$, respectively. This observation agrees with the results of Lasch et al. (10) who found, through an international ring-trial proficiency test panel, that identification results improved from $77 \%$ with a standard library and to $93.5 \%$ with the Robert Koch Institute supplementary library (10). Importantly, in-house libraries can be created if strains are available, which in the case of SSBA regulated pathogens is quite difficult. In Canada, SSBA work is limited, as only $0.2 \%$ of all regulated work involves activities with SSBAs, including both bacterial and viral RG3 and Risk Group 4 work (27). Thus, the distribution of the NML SSBA library to our Canadian public health laboratory partners is an important aspect of risk reduction.

Overall, MALDI-TOF MS is a powerful tool for signalling the presence of highly-pathogenic SSBA bacteria, but it is not a magic bullet. Diagnostic laboratories must consider augmentation of current practices with enhanced practices incorporating older tools such as Gram staining and colony morphology recognition, or moving sample preparation into a biological safety cabinet. The APHL recommendations state that sentinel laboratories should use the tube extraction method with filtration for suspected highly pathogenic bacteria and RG3 practices, including preparation in a biosafety cabinet. Written procedures for the recognition of the agents of bioterrorism and training should also be considered, in alignment with American Society for Microbiology and APHL sentinel guidelines, and bench cards illustrating features of high consequence bacteria can be incorporated into practice. Of note, all laboratories in Canada that reported exposure to an SSBA from MALDI-TOF MS use from 2015-2017 developed enhanced standard operating procedures, with triggers such as slow growth and observation of small gram-negative coccobacilli (8). Further incorporation of near neighbour warnings as detailed herein should further limit potential exposure incidents.

\section{Authors' statement}

DT - Performed laboratory technical work, data analysis and interpretation, drafted and revised the paper

$A D$ - Performed laboratory technical work, data analysis and interpretation, and revised the paper

KA and CC - Provided study conceptualization, data analysis and revised the paper 


\section{Competing interests}

None.

\section{Acknowledgements}

Thank you to the staff of the National Microbiology Laboratory, including Dr. AD Tyler and the Bioforensics Assay Development and Diagnostics (BADD) section, the Special Bacteriology group, the National Microbiology Laboratory (NML) Media core service and NML's Mass Spectrometry \& Proteomics core service.

\section{Funding}

This work was supported by the Public Health Agency of Canada, Government of Canada.

\section{References}

1. Doern CD, Butler-Wu SM. Emerging and Future Applications of Matrix-Assisted Laser Desorption Ionization Time-ofFlight (MALDI-TOF) Mass Spectrometry in the Clinical Microbiology Laboratory: A Report of the Association for Molecular Pathology. J Mol Diagn 2016;18(6):789-802. DOI PubMed

2. Patel R. MALDI-TOF MS for the diagnosis of infectious diseases. Clin Chem 2015 Jan;61(1):100-11. DOI PubMed

3. Ge MC, Kuo AJ, Liu KL, Wen YH, Chia JH, Chang PY, Lee MH, Wu TL, Chang SC, Lu JJ. Routine identification of microorganisms by matrix-assisted laser desorption ionization time-of-flight mass spectrometry: success rate, economic analysis, and clinical outcome. J Microbiol Immunol Infect 2017;50(5):662-8. DOI PubMed

4. Tran A, Alby K, Kerr A, Jones M, Gilligan PH. Cost Savings Realized by Implementation of Routine Microbiological Identification by Matrix-Assisted Laser Desorption Ionization-Time of Flight Mass Spectrometry. J Clin Microbiol 2015;53(8):2473-9. DOI PubMed

5. Keller PM, Bruderer V, Müller F. Restricted Identification of Clinical Pathogens Categorized as Biothreats by Matrix-Assisted Laser Desorption Ionization-Time of Flight Mass Spectrometry. J Clin Microbiol 2016;54(3):816. DOI PubMed

6. Dingle TC, Butler-Wu SM, Abbott AN. Accidental exposure to Burkholderia pseudomallei in the laboratory in the era of matrix-assisted laser desorption ionization-time of flight mass spectrometry. J Clin Microbiol 2014;52(9):3490-1. DOI PubMed
7. New York City Department of Health and Mental Hygiene. 2015 Alert \#15: Imported Brucellosis: Recent Laboratory Exposures Requiring Prophylaxis and Long-term follow up. New York (NY): NYCD of H and M; 2015.

https://www1.nyc.gov/assets/doh/downloads/pdf/han/alert/ imported-brucellosis.pdf

8. Pomerleau-Normandin D, Heisz M, Su M. Misidentification of Risk Group 3/Security Sensitive Biological Agents by MALDI-TOF MS in Canada: November 2015-October 2017. Can Commun Dis Rep 2018;44(5):110-5. DOI PubMed

9. Cunningham SA, Patel R. Importance of using Bruker's security-relevant library for Biotyper identification of Burkholderia pseudomallei, Brucella species, and Francisella tularensis. J Clin Microbiol 2013;51(5):1639-40. DOI PubMed

10. Lasch $P$, Wahab T, Weil S, Pályi B, Tomaso $H$, Zange $S$, Kiland Granerud B, Drevinek M, Kokotovic B, Wittwer M, Pflüger V, Di Caro A, Stämmler M, Grunow R, Jacob D. Identification of Highly Pathogenic Microorganisms by Matrix-Assisted Laser Desorption lonization-Time of Flight Mass Spectrometry: Results of an Interlaboratory Ring Trial. J Clin Microbiol 2015;53(8):2632-40. DOI PubMed

11. Tracz DM, Tyler AD, Cunningham I, Antonation KS, Corbett CR. Custom database development and biomarker discovery methods for MALDI-TOF mass spectrometry-based identification of high-consequence bacterial pathogens. J Microbiol Methods 2017;134:54-7. DOI PubMed

12. Tracz DM, Tober AD, Antonation KS, Corbett CR. MALDI-TOF mass spectrometry and high-consequence bacteria: safety and stability of biothreat bacterial sample testing in clinical diagnostic laboratories. J Med Microbiol 2018;67(3):341-6. DOl PubMed

13. Tracz DM, Antonation KS, Corbett CR. Verification of a Matrix-Assisted Laser Desorption lonization-Time of Flight Mass Spectrometry Method for Diagnostic Identification of High-Consequence Bacterial Pathogens. J Clin Microbiol 2016;54(3):764-7. DOI PubMed

14. Lasch P, Grunow R, Antonation KS, Weller SA, Jacob D. Inactivation techniques for MALDI-TOF MS analysis of highly pathogenic bacteria - A critical review. TrAC Trends Anal Chem. 2016;85:112-9. DOI

15. Achtman M, Zurth K, Morelli G, Torrea G, Guiyoule A, Carniel E. Yersinia pestis, the cause of plague, is a recently emerged clone of Yersinia pseudotuberculosis. Proc Natl Acad Sci USA 1999;96(24):14043-8. DOI PubMed

16. Chain PS, Carniel E, Larimer FW, Lamerdin J, Stoutland PO, Regala WM, Georgescu AM, Vergez LM, Land ML, Motin VL, Brubaker RR, Fowler J, Hinnebusch J, Marceau M, Medigue C, Simonet M, Chenal-Francisque V, Souza B, Dacheux D, Elliott JM, Derbise A, Hauser LJ, Garcia E. Insights into the evolution of Yersinia pestis through whole-genome comparison with Yersinia pseudotuberculosis. Proc Natl Acad Sci USA 2004;101(38):13826-31. DOI PubMed 
17. Gérôme $P$, Le Flèche $P$, Blouin $Y$, Scholz HC, Thibault FM, Raynaud F, Vergnaud G, Pourcel C. Yersinia pseudotuberculosis ST42 (O:1) Strain Misidentified as Yersinia pestis by Mass Spectrometry Analysis. Genome Announc 2014;2(3):e00435-14. DOI PubMed

18. Lasch $P$, Drevinek M, Nattermann H, Grunow R, Stämmler M, Dieckmann R, Schwecke T, Naumann D. Characterization of Yersinia using MALDI-TOF mass spectrometry and chemometrics. Anal Chem 2010;82(20):8464-75.

DOI PubMed

19. Seibold E, Maier T, Kostrzewa M, Zeman E, Splettstoesser W. Identification of Francisella tularensis by whole-cell matrix-assisted laser desorption ionization-time of flight mass spectrometry: fast, reliable, robust, and cost-effective differentiation on species and subspecies-levels. J Clin Microbiol 2010;48(4):1061-9. DOI PubMed

20. Antonation KS, Grützmacher K, Dupke $S$, Mabon $P$, Zimmermann F, Lankester F, Peller T, Feistner A, Todd A, Herbinger I, de Nys HM, Muyembe-Tamfun JJ, Karhemere S, Wittig RM, Couacy-Hymann E, Grunow R, Calvignac-Spencer S, Corbett CR, Klee SR, Leendertz FH. Bacillus cereus Biovar Anthracis Causing Anthrax in Sub-Saharan Africa-Chromosomal Monophyly and Broad Geographic Distribution. PLoS Negl Trop Dis 2016;10(9):e0004923. DOI PubMed

21. Ferreira $L$, Vega Castaño $S$, Sánchez-Juanes $F_{1}$ González-Cabrero S, Menegotto F, Orduña-Domingo A, González-Buitrago JM, Muñoz-Bellido JL. Identification of Brucella by MALDI-TOF mass spectrometry. Fast and reliable identification from agar plates and blood cultures. PLoS One 2010;5(12):e14235. DOI PubMed

22. Lista $F$, Reubsaet FA, De Santis $R$, Parchen RR, de Jong $A L$, Kieboom J, van der Laaken AL, Voskamp-Visser IA, Fillo S, Jansen HJ, Van der Plas J, Paauw A. Reliable identification at the species-level of Brucella isolates with MALDI-TOF-MS. BMC Microbiol 2011;11:267. DOI PubMed
23. Karger A, Melzer F, Timke M, Bettin B, Kostrzewa M, Nöckler K, Hohmann A, Tomaso H, Neubauer H, A Dahouk S. Interlaboratory comparison of intact-cell matrix-assisted laser desorption ionization-time of flight mass spectrometry results for identification and differentiation of Brucella spp. J Clin Microbiol 2013;51(9):3123-6. DOI PubMed

24. Sali M, De Maio F, Tarantino M, Garofolo G, Tittarelli M, Sacchini L, Zilli K, Pasquali P, Petrucci P, Marianelli C, Francia M, Sanguinetti M, Adone R. Rapid and safe one-step extraction method for the identification of Brucella strains at genus and species-level by MALDI-TOF mass spectrometry. PLoS One 2018;13(6):e0197864. DOI PubMed

25. Sogawa K, Watanabe M, Sato K, Segawa S, Miyabe A, Murata S, Saito T, Nomura F. Rapid identification of microorganisms by mass spectrometry: improved performance by incorporation of in-house spectral data into a commercial database. Anal Bioanal Chem 2012;403(7):1811-22. DOI PubMed

26. Christensen JJ, Dargis R, Hammer M, Justesen US, Nielsen XC, Kemp M; Danish MALDI-TOF MS Study Group. Matrix-assisted laser desorption ionization-time of flight mass spectrometry analysis of Gram-positive, catalase-negative cocci not belonging to the Streptococcus or Enterococcus genus and benefits of database extension. J Clin Microbiol 2012 May;50(5):1787-91. DOI PubMed

27. Pomerleau-Normandin D, Heisz M, Tanguay F. Surveillance of laboratory exposures to human pathogens and toxins: Canada 2017. Can Commun Dis Rep 2018 Nov;44(11):297-304. DOI PubMed 